\title{
Acute Appendicitis Associated with CT Intraluminal Hyperattenuation
}

\author{
Mohsen Kamel Arid \\ Department of Radiology Benha Faculty of Medicine, Benha, Egypt \\ Email: mkarid2002@yahoo.com
}

How to cite this paper: Arid, M.K. (2021) Acute Appendicitis Associated with CT Intraluminal Hyperattenuation. Open Jour nal of Radiology, 11, 1-10.

https://doi.org/10.4236/ojrad.2021.111001

Received: December 8, 2020

Accepted: February 5, 2021

Published: February 8, 2021

Copyright (c) 2021 by author(s) and Scientific Research Publishing Inc. This work is licensed under the Creative Commons Attribution International License (CC BY 4.0).

http://creativecommons.org/licenses/by/4.0/

\begin{abstract}
Introduction: The appendix is identified as blind ending tubular structure arising from caecum and has variable intraluminal contents and position. Acute appendicitis is one of the common indications for emergency imaging studies. Aim: To describe the importance of appendix hyperattenuation and densities. Material and Methods: Contrast enhanced computed tomography images of abdomen from 120 patients with surgically/pathological proven acute appendicitis, were examined retrospectively. The images were reviewed in axial, coronal and sagittal reformations for assessing the intraluminal contents (hyperdensity and appendicolith), maximum transverse diameter and single wall thickness of appendix, periappendiceal fat stranding and other parameters related to acute appendicitis. In addition, reviewing 100 pre- and post-contrast $\mathrm{CT}$ scans of other abdominal conditions as a control group for documenting hyperdense appendix, appendicolith and other signs of appendicitis. Results: The hyperdense appendix sign was found in 5 patients in our study, not found in any patient of the control group ( $\mathrm{P}$ value $=0.039$, is statistically), appendicolith was found in $25 \%$ in patients with acute appendicitis, in $3 \%$ in control group (statistically significant, $\mathrm{p}<0.0001$ ). Conclusion: The hyperdense appendix and appendicolith have strong association with acute appendicitis in the appropriate clinical setting.
\end{abstract}

\section{Keywords}

Unenhanced CT, Appendicolith, Appendicitis, Hyperdense Appendix

\section{Introduction}

Acute appendicitis is one of the most common surgical emergencies worldwide affecting approximately $7 \%$ of the general population in a lifetime [1]. Laparoscopic appendectomy is widely gaining acceptance as the treatment of choice of 
patients with acute appendicitis [1].

Computed tomography (CT) is the imaging modality of choice for suspected acute appendicitis, due to its high sensitivity and specificity [2]. CT is more accurate than ultrasonography (USG) as CT is less operator-dependent. [3]. Also, patient factors like obesity, overlying gas-filled bowel loops and differences in positions of appendix may pose serious challenges in visualizing abnormal appendix with ultrasound [4].

Hyperdensity material (hyperattenuation) of the appendix has a common cause, like appebcolith, and other less common causes can be seen.

The obstruction of the lumen triggers the inflammation process in the appendix. The most common pathologies associated with lumen obstruction are lymphoid hyperplasia, fecalith, stricture and appendicolith. Stasis and bacterial colonization in the appendix lumen result in appendicitis.

The appendicolith is formed by firm, dense stool and mineral deposits. It is also known as appendiceal calculi, appendiceal enterolith or appendicular lithiasis. Appendicoliths are usually seen in pediatric populations and young adults. They are detected more frequently in men [5].

Blood clot resulting from recent hemorrhage is well known to appear as hyperdense focus presented on non contrast CT scans. Mucosal hemorrhage secondary to ischemia is often found in pathology examination of acute appendicitis [6].

Valluru, et al. in their CT study of 50 elderly patients of schistosomal associated appendicitis found larger appendicular diameter, appendicular wall calcifications along with sigmoid colon, and cecal calcifications, in addition to perforation or abscess formation [7].

Fataar and Satyanath radiographically detected appendiceal calcification in 25 patients infected by schistosomiasis [8].

Herper, et al. described large amount of unexpected high-attenuation intraluminal material. By the CT scan, appendiceal enlargement and associated free fluid are also seen. With further history, this was thought to be most likely retained bismuth from over-the-counter medicine ingestion and ultimately an appendectomy was performed [9].

Barium appendicitis is a rare complication of barium meal or enema studies. Although barium sulphate is inert and not harmful to the mucosa, 3 cases have been reported where appendicitis developed long after barium studies and were tagged as barium induced appendicitis [10].

On the basis of CT findings, Stengel, et al. [11] classified the likelihood of appendicitis into five grades: grade 1 , definitely not appendicitis; grade 2 , nonvisualization of appendix with no secondary signs of inflammation; grade 3, equivocal; grade 4 , probable; and grade 5 , high possibility of or compatible with appendicitis. Using this classification, only grade 1 or 5 helps provide a definitive conclusion for surgeons, and the remaining grades suggest weaker or stronger clues regarding appendicitis. 


\section{Material and Methods}

The study was conducted in a private hospital, in Abu Dhabi, Radiology Department.

120 patients with surgically or pathologically proven acute appendicitis who underwent pre- and post-enhanced CT of the abdomen, over one year period, from March 2016 to march 2017. The images were retrospectively reviewed on work stations. Negative appendectomy patients were excluded. 100 control group patients with CT-abdomen done for other conditions, were also retrospectively reviewed for search of hyperdense appendix or appendicolith or signs of inflammation of the appendix.

All patients underwent unenhanced CT abdomen. Intravenous and/or enteric positive contrast scans were done in majority of patients (few patients refused contrast or have allergy), using Philips Brilliance 64 CT scanner.

The study included 120 patients, out of which 79 were males and 41 were females. Age of subjects ranged from 15 to 65 years.

\section{Image Analysis}

Using Philips CT-work station, the images were retrospectively reviewed by two radiologists who reached a decision by consensus.

Radiological diagnosis of appendicitis was made when appendiceal dilatation was of $>6 \mathrm{~mm}$ with any of the following additional features were present: appendiceal wall thickness of $>3 \mathrm{~mm}$, peri-appendiceal fat stranding, peri-appendiceal free fluid or heterogenous appendiceal wall enhancement (in case of contrastenhanced CT imaging), Khan, et al., 2019 [12]. In addition to, the presence of an appendicolith or hyperdense materials in the appendix, intraluminal fluid, appendiceal gas, and right lower quadrant lymphadenopathy. Appendicolith was defined as an intraluminal lesion that demonstrated a high density, similar to that of the adjacent bone [13]. Hyperdense material in the appendix was defined as Appendix, that showed segmental, focal or diffuse high-attenuation when compared with the adjacent cecal wall on precontrast CT [6]. Lymphadenopathy was defined as an enlarged node measuring $>8 \mathrm{~mm}$ at its smallest diameter [14].

Hyperdensity in non dilated appendix fall in grade 3 or 4 , equivocal or probable appendicitis according to strengel, et al. [11].

The appendix was having variable positions from subcaecal, retrocolic/retrocaecal, subhepatic, pelvic-extending to the pelvis; midline-extending to the midline, the location was not significant in our study.

Statistical analyses were performed using SPSS software, the variables were compared using the Chi-square test between the true appendicitis and control groups, and significance was assigned at a $\mathrm{P}$-value of $<0.05$.

\section{Results}

The appendix was visualized in all the 120 patients included. Hyperdense appendix was found in 5 patients with acute appendicitis (4.1\%), 4 patients has diffuse luminal hyperdensity of normal caliber appendix (Figure 1 and Figure 2 ), one patient had focal hyperdensity in slightly dilated appendix (Figure 3). No 
patient in the control group has this sign (0\%), the difference was statistically significant $(\mathrm{P}=0.039)$ (Table 1). Appendicolith was found in appendicitis group in 30 patients (25\%), the appendicoliths, seen in this group are more than $5 \mathrm{~mm}$ and associated with dilated appendix (Figure 4). Three cases of appendicoliths in the control group (3\%), not associated with dilated appendix (Figure 5), this is a statistically significant $(\mathrm{p}=0.000005)$ (Table 1$)$. The appendiceal dilation was seen in 90 cases (75\%) in the appendicitis group, 3 cases (3\%) had an enlarged appendix in the control group (Figure 6). Periappendiceal fat inflammation or fat stranding was observed in 96 patients (80\%) in the appendicitis group, and in 3 patients (3\%) in the control group. Appendiceal wall thickening was observed in 101 patients $(84.2 \%)$ in the appendicitis group and in 3 patients (3\%) in the control group. Appendiceal wall enhancement was seen in 95 patients $(81.7 \%)$ in appendicitis group (Figure 7). Mesenteric lymphadenopathy was observed in $20 \mathrm{pa}-$ tients $(16.7 \%)$ in the appendicitis group and 4 patients $(4 \%)$ in the control group.

Table 1. Comparison of the percentage and significance of the CT findings in both groups.

\begin{tabular}{cccc}
\hline CT finding & $\begin{array}{c}\text { Proven appendicitis } \\
(\mathbf{N}=\mathbf{1 2 0})\end{array}$ & $\begin{array}{c}\text { Control group } \\
\mathbf{( N = 1 0 0 )}\end{array}$ & P-value \\
\hline Hyperdense appendix & $5(4.1 \%)$ & $0(0 \%)$ & 0.039 \\
Dilated appendix & $90(75 \%)$ & $3(3 \%)$ & 0.0001 \\
Thickened wall & $101(84.2 \%)$ & $3(3 \%)$ & 0.0001 \\
Fat stranding & $96(80 \%)$ & $3(3 \%)$ & 0.0001 \\
Fluid collection & $24(20 \%)$ & $2(2 \%)$ & 0.00004 \\
Mesenteric LNs & $20(16.7 \%)$ & $4(4 \%)$ & 0.0027 \\
Appendicolith & $30(25 \%)$ & $3(3 \%)$ & 0.000005 \\
Wall enhancement & $98(81.7 \%)$ & & \\
Periappendicular abscess & $8(6.7 \%)$ & & \\
\hline
\end{tabular}

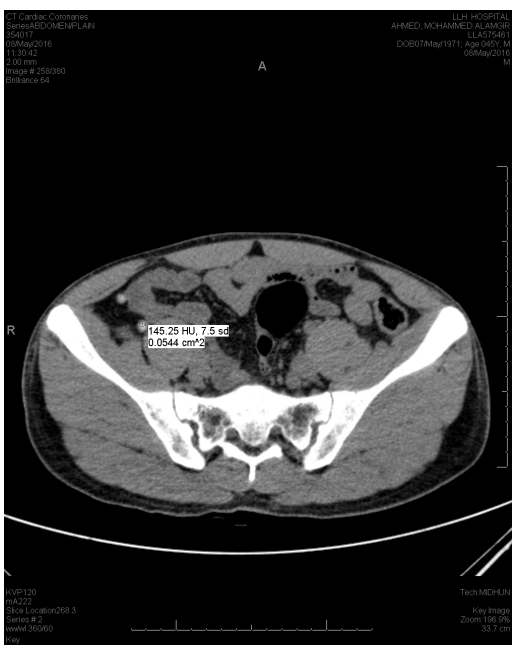

(a)

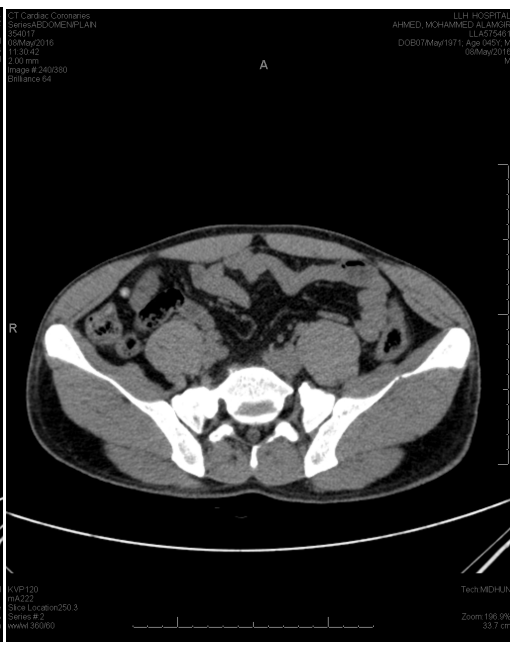

(b)

Figure 1. Hyperdense, non dilated appendix in axial non contrast CT abdomen, $(\mathrm{a}, \mathrm{b})$ in a 45 -year-old male. 


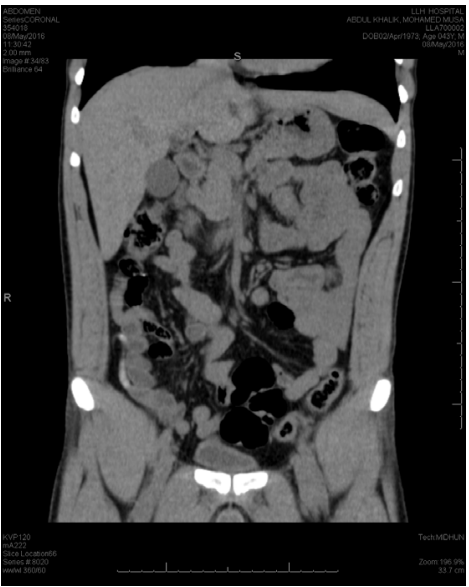

(a)

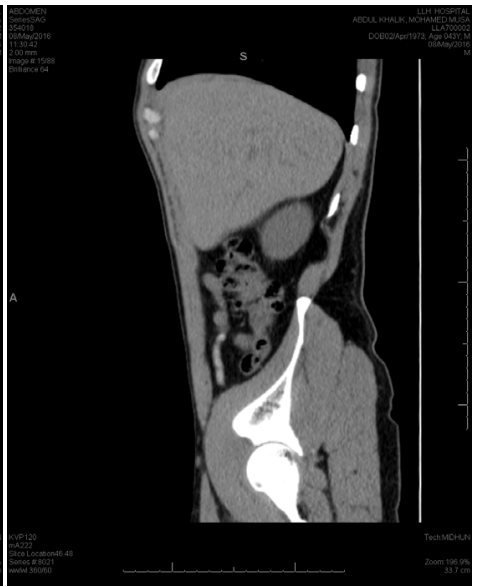

(b)

Figure 2. Diffuse Hyperdense, non dilated, appendix, in sagittal and coronal non contrast CT of Abdomen in 43-year-old male.

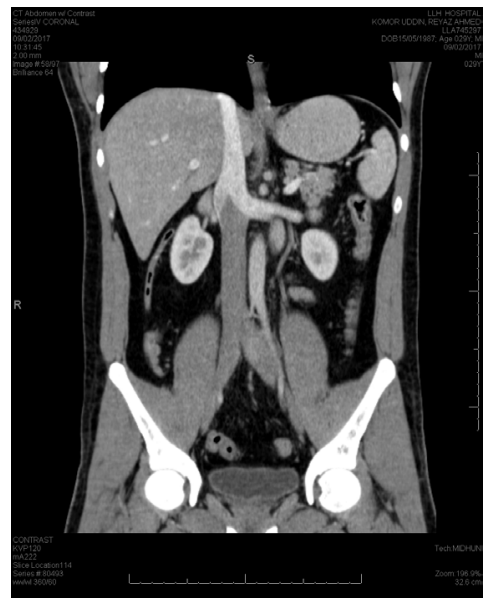

Figure 3. Focal hyperdensity of dilated subhepatic, appendix in contrast CT of 29 yo male.

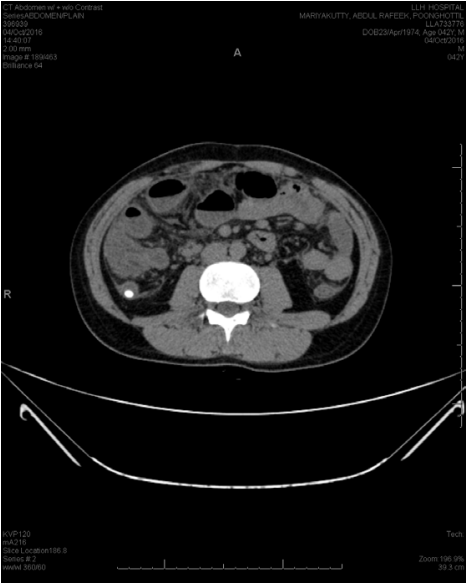

(a)

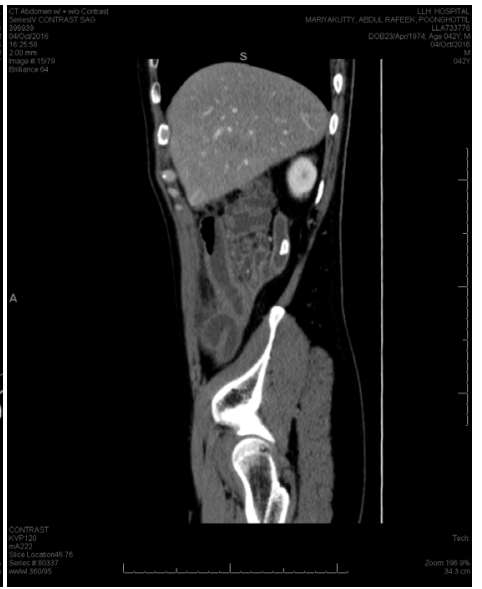

(b)

Figure 4. Appendicolith with dilated appendix in axial, and sagittal non-contrast CT scan of 42 yo male. 


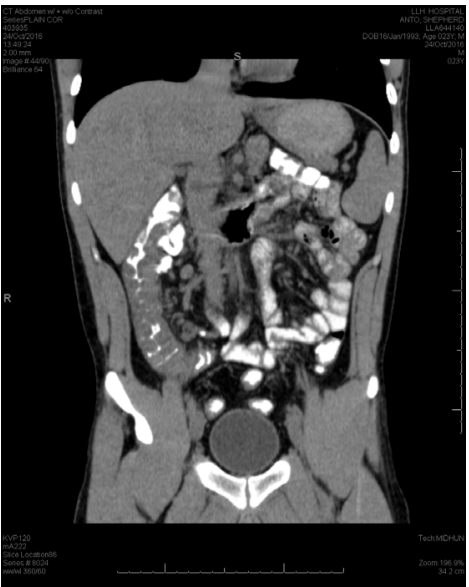

(a)

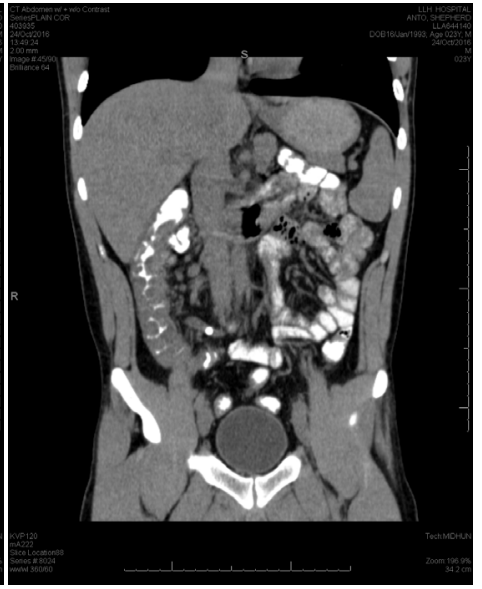

(b)

Figure 5. Appendicolith in non-dilated appendix in coronal contrast CT abdomen, (a, b). In 23 yo male patient with pseudo-membranous colitis.

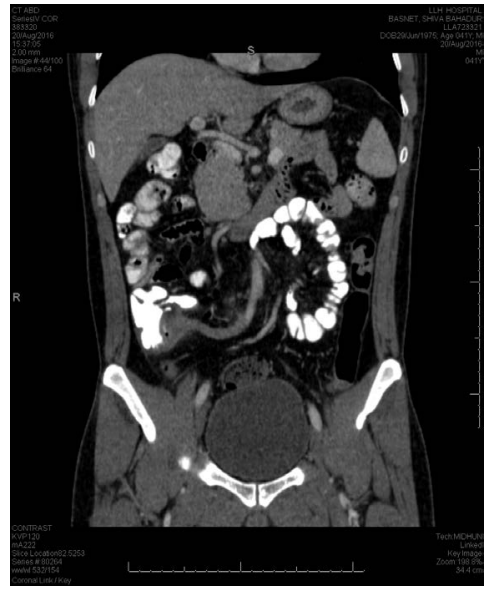

(a)

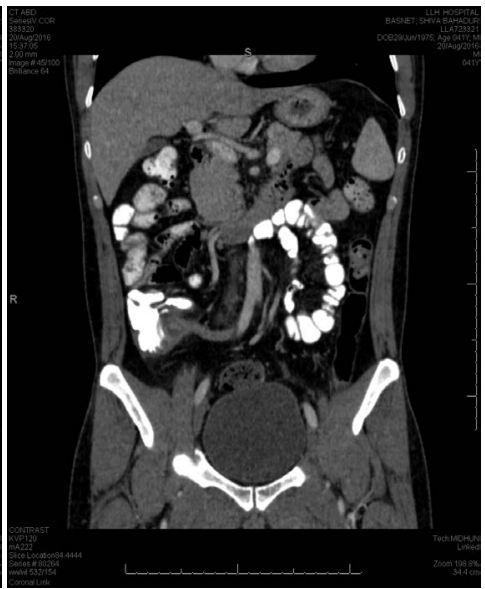

(b)

Figure 6. Appendicitis. Dilated appendix with cecal indentation, arrow sign. In coronal contrast CT scan of 41 yo male.

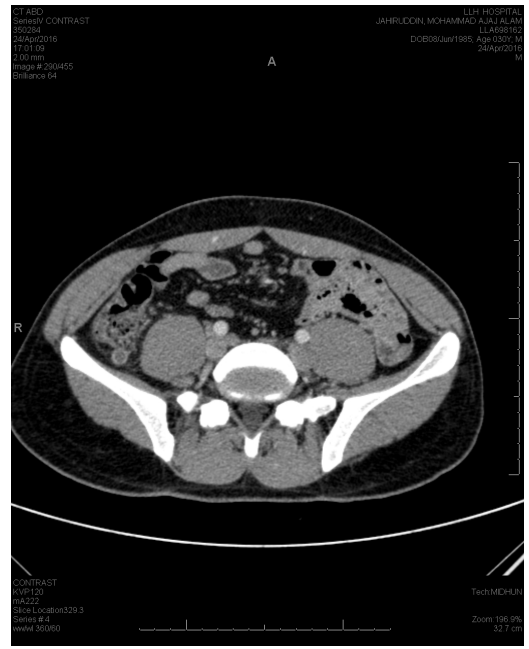

Figure 7. Contrast enhanced wall of a dilated appendix in contrast CT of 30 yo male. 


\section{Discussion}

A hyperdense appendix sign was found in 61 of 183 (33\%) patients of acute appendicitis. On the other hand, the sign was seen in only two (2\%) of the 88 patients in control group. The hyperdense appendix sign on unenhanced CT is seen in about $33 \%$ of patients with acute appendicitis. The false-positive rate is very low, rendering it a very useful sign for diagnosis of acute appendicitis [6].

In our study we found diffuse hyperdense appendix in 5 of 120 patients (4.1\%), much less than was reported by Ng, et al. 2007 [6] who reported 61/183 patients (33\%) with hyperdense appendix, this discrepancy may be due to the way he assessed hyperdense appendix whether focal or diffuse, faint or dense.

In our study, if appendicolith patients combined with the hperdense appendix cases (some authors called it hyperdense mucosal sign, Kim and Moon [15]), we have 35 out 120 patients (29.1\%) have acute appendicitis with appendiceal hyper attenuation.

$\mathrm{Ng}$, et al. 2007 [6] explained this hperdense appendix sign is likey, due to mucosal hemorrhage secondary to ischemia is often found in pathology examination of acute appendicitis. Therefore, just as in ischemic bowel disease, where hyper-attenuated bowel wall may be present on unenhaced CT scans, a similar finding might appear in appendicitis. In addition, the small lumen of the appendix may accentuate the hyperdensity resulting from formation of recent blood clot [6].

An appendicolith was seen in $65 \%$ of CT scans of children with proven appendicitis and in $14 \%$ of children with abdominal pain but without appendicitis (Lowe, et al., 2000) [16].

As a nidus for appendicolith, the prevelance of facecolith has been reported as $3 \%$ in the population by Jones, et al. They also stated that the low-fiber diet has been associated with increased risk of fecalith formation [17].

Appendicoliths are seen in about $10 \%$ of patients with acute appendicitis [18]. Appendicoliths are present in one third of patients with appendicitis. Although associated with appendicitis, appendicoliths are not diagnostic and have low specificity as isolated findings because they are commonly present in asymptomatic subjects. Appendicoliths may have prognostic importance, however, because their presence increases the likelihood of appendiceal perforation [19].

Previous studies reported that appendicolith is well-defined hyperdense non-enhancing structure that strongly associated with advanced appendicitis and is a risk factor for perforation and necrosis [20].

Larger size of appendicoliths and its more proximal location has also been found to be associated with complicated appendicitis [21]. Perforations may occur due to high luminal pressure from ongoing obstruction leading to ischemia, gangrene and ultimately rupture of the appendix [21].

Significantly greater proportion of patients in the acute appendicitis group had appendicolith at the base in acute appendicitis [(33\%) vs. (15\%), $\mathrm{p}<0.001]$ 
and appendicolith diameter of $5 \mathrm{~mm}$ or more in acute appendicitis [(69\%) vs. (13\%), p < 0.001], khan, et al., 2019 [12]. Their results showed that appendicoliths that were larger in size and multiple in number were associated with acute appendicitis [12].

Kim and Moon [15] reported appendicoliths in 8 of 39 patients in his pediatric study (20.1\%), also we reported in our study appendicoliths in 30 patients with appendicitis, 30 out of 120 patients $(25 \%)$ in adult population.

Narayan and Joseph suggest that a diameter of $6 \mathrm{~mm}$ may not be a reliable cut off to predict appendicitis in the absence of other signs [22].

The reported diameter of a normal appendix at CT is $6 \mathrm{~mm}$ in short-axis diameter as the upper limit of normal [23]. However normal appendiceal diameter ranging from $6-11 \mathrm{~mm}$ in CT has been shown by recent studies [24].

Another study added that increased appendiceal caliber alone is not a reliable indicator of appendicitis and must be considered alongside the patient's clinical history and other imaging findings [25].

We reported in our study appendiceal dilation or enlargement in 90 out of 120 patients (75\%), this is in accordance with Kim \& Moon who reported appendiceal enlargement in 29 of 39 patients (74.4\%) [15].

Recent articles have suggested that wall thickness of the appendix is a more reliable measurement than appendiceal diameter [26].

We demonstrated thickened appendiceal wall in 101 patients out of 120 (84.2\%) but Kim \& Moon [15] in their study reported it in 39/39 (100\%).

Periappendiceal stranding in 96 out 120 patients (80\%) in our study, Kim and Moon [15] had this sign in $32 / 39$ (82.1\%) and we found appendiceal wall enhancement in 98 out of 120 of our patients $(81.7 \%)$ the same as Kim \& Moon had $32 / 39(82.1 \%)$ in their study.

Lymphadenopathy in our study was found in 20/120 (16.7\%). In Kim and Moon [15], it was 38/39 (97.4\%), higher than in our study because they studied pediatric patients.

In a recent study, by Choi, et al. [27] concluded that appendiceal enlargement, appendiceal wall thickening, periappendiceal fat stranding, and appendiceal wall enhancement were significantly more associated with acute appendicitis than with other findings.

\section{Conclusion}

Our study concluded that Hyperdense appendix and appendicoliths are associated with $29.1 \%$ of cases of acute appendicitis. We suggest more studies about the significance of diffuse high attention of the normal size appendix in non contrast CT abdomen.

\section{Conflicts of Interest}

The author declares no conflicts of interest regarding the publication of this paper. 


\section{References}

[1] Singh, A.K., Hahn, P.F., Gervais, D., Vijayraghavan, G. and Mueller, P.R. (2008) Dropped Appendicolith: CT Findings and Implications for Management. American Journal of Roentgenology, 190, 707-711. https://doi.org/10.2214/AJR.07.2917

[2] Ghiatas, A.A. and Kritikos, N. (2004) CT of Appendicitis. In: Baert, A.L., Sartor, K. and Chapman, A.H., Eds., Radiology and Imaging of the Colon, Springer-Verlag, Berlin, Heidelberg, 157-163. https://doi.org/10.1007/978-3-642-18834-3 15

[3] Bursali, A., Arac, M., Oner, Y.A., Celik, H., Eksioglu, S. and Gumus, T. (2005) Evaluation of the Normal Appendix at Low-Dose Non-Enhanced Spiral CT. Diagnostic and Interventional Radiology, 11, 45-50.

[4] Akay, H.O., Akpinar, E., Ozmen, C.A., Ergun, O. and Haliloglu, M. (2007) Visualization of the Normal Appendix in Children by Non-Contrast MDCT. Acta Chirurgica Belgica, 107, 531-534. https://doi.org/10.1080/00015458.2007.11680116

[5] Nitecki, S., Karmeli, R. and Sarr, M.G. (1990) Appendiceal Calculi and Fecaliths as Indications for Appendectomy. Surgery, Gynecology \& Obstetrics, 171, 185-188.

[6] Ng, S.P., Cheng, S.M., Yang, F.S., et al. (2007) Hyperdense Appendix on Unenhanced CT: A Sign of Acute Appendicitis. Abdominal Radiology, 32, 701-704.

https://doi.org/10.1007/s00261-007-9176-6

[7] Valluru, B., Zhou, Z., Sah, D., et al. (2020) Analysis of CT Characteristics in the Diagnosis of Schistosoma japonicum Associated Appendicitis with Clinical and $\mathrm{Pa}$ thological Correlation: A Diagnostic Accuracy Study. Japanese Journal of Radiology, 38, 178-191. https://doi.org/10.1007/s11604-019-00905-4

[8] Fataar, S. and Satyanath, S. (1986) The Radiographic Evaluation of Appendiceal Calcification Due to Schistosomiasis. The American Journal of Tropical Medicine and Hygiene, 35, 1157-1162. https://doi.org/10.4269/ajtmh.1986.35.1157

[9] Harper, R., Friedman, B.T. and Strote, J. (2016) Missed Appendicitis: Did Unexpected Intraluminal Densities Play a Role? Case Reports, bcr2016216241.

[10] Urade, M. and Shinbo, T. (2012) Barium Appendicitis 1 Month after a Barium Meal. International Surgery, 97, 296-298. https://doi.org/10.9738/CC160.1

[11] Stengel, J.W., Webb, E.M., Poder, L., Yeh, B.M., Smith-Bindman, R. and Coakley, F.V. (2010) Acute Appendicitis: Clinical Outcome in Patients with an Initial FalsePositive CT Diagnosis. Radiology, 256, 119-126.

https://doi.org/10.1148/radiol.10091229

[12] Khan, M.S., Chaudhry, M.B.H., Shahzad, N., Khan, M.S., Wajid, M., Memon, W.A. and Alvi, R. (2019) The Characteristics of Appendicoliths Associated with Acute Appendicitis. Cureus, 11, e5322.

[13] Searle, A.R., Ismail, K.A., Macgregor, D. and Hutson, J.M. (2013) Changes in the Length and Diameter of the Normal Appendix throughout Childhood. Journal of Pediatric Surgery, 48, 1535-1539. https://doi.org/10.1016/j.jpedsurg.2013.02.035

[14] Karmazyn, B., Werner, E.A., Rejaie, B. and Applegate, K.E. (2005) Mesenteric Lymph Nodes in Children: What is Normal? Pediatric Radiology, 35, 774-777. https://doi.org/10.1007/s00247-005-1462-2

[15] Kim, Y. and Moon, S. (2016) Inconclusive Abdominal CT Findings in Pediatric Patients with Appendicitis: Lessons from Negative Appendectomy Cases. Reports in Medical Imaging, 9, 1-6. https://doi.org/10.2147/RMI.S101126

[16] Lowe, L.H., Penney, M.W., Scheker, L.E, et al. (2000) Appendicolith Revealed on CT in Children with Suspected Appendicitis: How Specific is It in the Diagnosis of Appendicitis? American Journal of Roentgenology, 175, 981-984. 
https://doi.org/10.2214/ajr.175.4.1750981

[17] Jones, B.A., Demetriades, D., Segal, I. and Burkitt, D.P. (1985) The Prevalance of Appendiceal Fecaliths in Patients with and without Appendicitis. A Comparative Study from Canada and South Africa. Annals of Surgery, 202, 80-82.

https://doi.org/10.1097/00000658-198507000-00013

[18] Teke, Z., Kabay,B., Erbiş, H. and Tuncay, O.L. (2008) Appendicolithiasis Causing Diagnostic Dilemma: A Rare Cause of Acute Appendicitis (Report of a Case). Ulusal Travma Ve Acil Cerrahi Dergisi, 14, 323-325.

[19] Leite, N.P., Pereira, J.M., Cunha, R., Pinto, P. and Sirlin, C. (2005) CT Evaluation of Appendicitis and Its Complications: Imaging Techniques and Key Diagnostic Findings. American Journal of Roentgenology, 185, 406-417. https://doi.org/10.2214/ajr.185.2.01850406

[20] Avanesov, M., Wiese, N.J., Karul, M., Guerreiro, H., Keller, S., Busch, P., et al. (2018) Diagnostic Prediction of Complicated Appendicitis by Combined Clinical and Radiological Appendicitis Severity Index (APSI). European Radiology, 28, 3601-3610. https://doi.org/10.1007/s00330-018-5339-9

[21] Ishiyama, M., Yanase, F.,Taketa, T., Makidono, A., Suzuki, K., Omata, F. and Saida, Y. (2013) Significance of Size and Location of Appendicoliths as Exacerbating Factor of Acute Appendicitis. Emergency Radiology, 20, 125-130. https://doi.org/10.1007/s10140-012-1093-5

[22] Varun, N. and Sajin, G.J. (2019) Analysis of Morphology of Normal Appendix Using Contrast Enhanced CT Abdomen. International Journal of Contemporary Medicine Surgery and Radiology, 4, C40-C43.

[23] Stroman, D.L., Bayouth, C.V., Kuhn, J.A., et al. (1999) The Role of Computed Tomography in the Diagnosis of Acute Appendicitis. The American Journal of Surgery, 178, 485-489. https://doi.org/10.1016/S0002-9610(99)00223-8

[24] Tamburrini, S., Brunetti, A., Brown, M., Sirlin, C.B. and Casola, G. (2005) CT Appearance of the Normal Appendix in Adults. European Radiology, 15, 2096-2103. https://doi.org/10.1007/s00330-005-2784-Z

[25] Eng, K.A., Abadeh, A., Ligocki, C., Lee, Y.K., Moineddin, R., Adams-Webber, T., et al. (2018) Acute Appendicitis: A Meta-Analysis of the Diagnostic Accuracy of US, CT, and MRI as Second-Line Imaging Tests after an Initial US. Radiology, 288, 717-727. https://doi.org/10.1148/radiol.2018180318

[26] Athanasiou, C., Lockwood, S. and Markides, G.A. (2017) Systematic Review and Meta-Analysis of Laparoscopic Versus Open Appendicectomy in Adults with Complicated Appendicitis: an Update of the Literature. World Journal of Surgery, 41, 3083-3099. https://doi.org/10.1007/s00268-017-4123-3

[27] Choi, D., Park, H., Lee, Y.R., et al. (2003) The Most Useful Findings for Diagnosing Acute Appendicitis on Contrast-Enhanced Helical CT. Acta Radiologica, 44, 574-582. https://doi.org/10.1046/j.1600-0455.2003.00146.x 